International Journal of Information Technology, Control and Automation (IJITCA) Vol.3, No.2, April 2013

\title{
DEVELOPMENT OF AN AUTONOMOUS Y4 COPTER
}

\author{
Md. Shafayat Hossain, Ariyan M Kabir, Pratyai Mazumder, Ahmedullah Aziz, \\ Masudul Hassan Quraishi and Pran Kanai Saha \\ Dept. of Electrical and Electronic Engineering, Bangladesh University of Engineering \\ and Technology, Dhaka, Bangladesh \\ rumi3.1416@gmail.com
}

\begin{abstract}
The developed y4 copter is an Unmanned Aerial Vehicle with autonomous subsystems which reports the fully automated y4 copter for the first time. Automation is implemented through maintaining the desired roll, pitch and yaw therefore the flight stability as well as in case of controlling elevation. An improved and efficient algorithm that uses Quaternion is implemented to determine Euler angles avoiding Gimbal lock in case of developing an inertial measurement unit. It has a self-stabilization system powered by Proportional Integral Derivative control system which is computationally affordable by an ordinary 8 bit microcontroller capable of performing only integer operations. A remote is designed to communicate with the y4 copter from the base station using XBee transceiver modules which come with secured communication with long range. The developed system demonstrates simple design offering lower cost and secured means of control.
\end{abstract}

\section{KEYWORDS}

Automation, PID controller, Quaternion, Unmanned Aerial Vehicle

\section{INTRODUCTION}

Military surveillance, rescue, aerial photography, spying, information collection, crowd management and many other important applications characterize the importance of autonomous as well as fully-controllable aerial vehicle which is yet to be popularized in Indian subcontinent. Design of an unmanned aerial vehicle (UAV) involves several complexities like aerodynamic complexities, choice of number of rotors, mechanical difficulties, controlling processor, control algorithm and control system designing etc. With the increase in the number of rotors, flight stability increases and so does the complexity resulting in requirement of high processing power. An y4 copter has 4 rotors with controlling complexity just within the reach of an 8 bit microcontroller. Moreover this structure is free from servo mechanism required for controlling pitch. Proportional Integral Derivative (PID) control is implemented for flight stabilization which is the best choice for a control system to be implemented in an 8 bit microcontroller. A custom remote is implemented to control the $\mathrm{y} 4$ copter with XBee modules which provides long-range yet secured data transmission.

Commonly y 4 copters have been implemented by hobbyists using quad rotor control system with a rotor configuration shown in Fig. 1. This paper represents an y4 copter with different rotor configuration as shown in Fig. 2 with a unique control system. This rotor configuration is suggested in our previous work [1]. No reference of any previous work other than [1] on control system designing for $\mathrm{y} 4$ copter has been reported whatsoever. This paper reports the total automation of the y4 copter completing the work reported in [1]. 
International Journal of Information Technology, Control and Automation (IJITCA) Vol.3, No.2, April 2013

\section{Aerodynamic Design}

\subsection{Rotor Position and Configuration}

Most commonly used rotor configuration for y4 copter is depicted in Fig. 1.

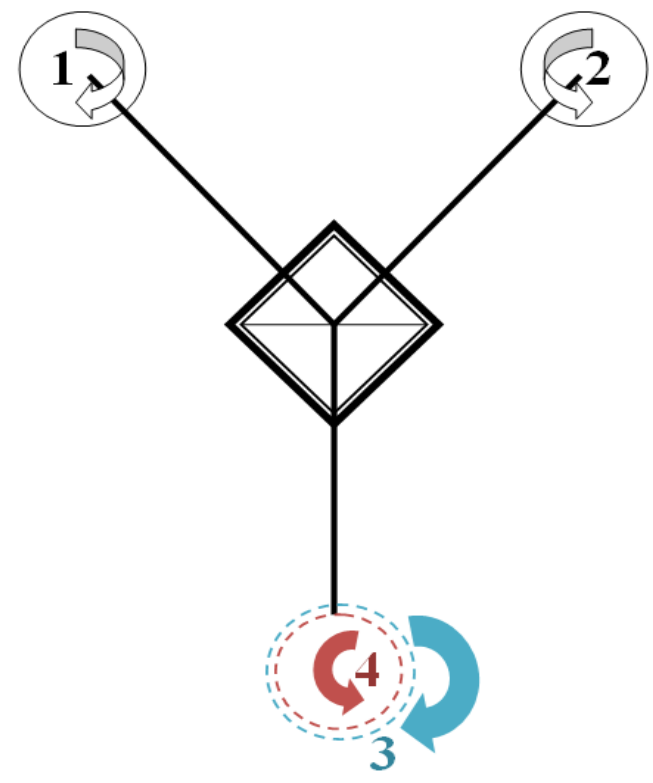

Figure 1. Rotor position and orientation of conventional Y4 copters

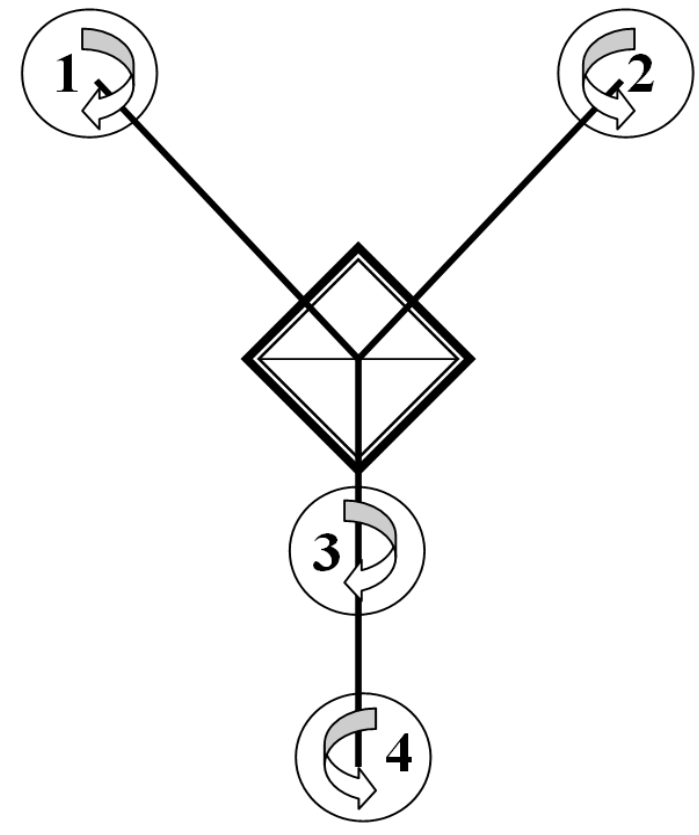

Figure 2. Rotor position and orientation of the designed Y4 copter 
Here rotor 3 and 4 are placed back to back which is mechanically complicated. For mechanical simplicity a new rotor configuration is introduced as an experimentation in [1] which is shown in Fig. 2. This configuration also introduces a different flight control mechanism.

\subsection{Flight Basics}

The concepts of air pressure are utilized in aeronautical designs and air pressure is exploited to create a lift force. Few core concepts of pressure are discussed in this sub-section and some necessary assumptions are made [2].

The first concept is the air pressure. When designing a system for flight, pressure ratio is used commonly relating the current pressure with the pressure at sea level. Though there exists no direct equation between air pressure and altitude, a working relation can be established.

The next basic concept is temperature. It is directly related with pressure. This effect is also expressed through ratios.

Density, the next basic concept, also contributes to the aeronautics of the y4 copter. Density, in terms of air is expressed in the following equation:

$$
\text { Density equation: } \rho=P / R T
$$

In this equation, $\mathrm{R}$ is the gas constant, $\mathrm{P}$ denotes pressure and $\mathrm{T}$ is the temperature. Thus, a similar relationship can be described for the density ratio.

The final basic concept to consider in preparing wing and craft design is air viscosity. This constant becomes apparent at speeds close to or exceeding the speed of sound. At slower speeds, viscosity becomes insignificant in the presence of calmer winds.

Ideal conditions for viscosity are assumed in the design of the $\mathrm{y} 4$ copter for simplicity. It is also assumed that the pressure is relatively equal to sea level i.e. the pressure ratio is at $0 \mathrm{~km}$ altitude as pressure does not change significantly for at about $2 \mathrm{~km}$ above sea level.

Airspeed at a cross-section, or the continuity and Bernoulli's equations also involve the effect of pressure. By the continuity equation, the pressure of a fluid passing through an area at a constant velocity can be described as:

$$
\text { Continuity equation: } \mathrm{pAV}=\mathrm{k}
$$

Here, $\mathrm{p}$ is static pressure, $\mathrm{A}$ is the measured cross-sectional area, and $\mathrm{V}$ is the velocity of the fluid. Bernoulli adopted it to describe the effects of dynamic pressure relating air density to air speed which is given as:

$$
\text { Dynamic Pressure equation: } \mathrm{q}=1 / 2 \times \rho \mathrm{V}^{2}
$$

In this case, $\rho$ is the air density and $\mathrm{V}$ is the speed of the air. This equation expedites calculation of the necessary tangential airspeed travelling through an airfoil responsible for the generation of required amount of lift. By this relation, lift is related to angular speed as well as centrifugal force. 


\subsection{Lift and Flight Stabilization}

To maintain flight stability, two forces must be considered: Lift force and the Magnus effect. Lift force is the most important force pertaining to flight which is based on the upward vertical component acting on an airfoil's aerodynamic center. The force reacting to the thrust of the airfoil moves perpendicularly to the airfoil, which can be represented as a vertical and horizontal force. The horizontal vector is termed as the drag force (as the name suggests, it moves opposite to the thrust). Lift is related to air density by the following equation:

$$
\text { Lift equation: } \mathrm{L}=\mathrm{C}_{\mathrm{d}} \mathrm{qS}
$$

Here, $C_{d}$ is the lift coefficient, $q$ denotes the dynamic pressure and $S$ represents the area of the airfoil. For most airfoils, measurement is done at the center of pressure, which is usually located at the center of the chord of the blade.

In terms of measurement of the lift for a helicopter, the propeller speed is considered rather than the thrust as the propeller, which is with few exceptions virtually vertical, is, in this case, operating on a horizontal plane. The centrifugal force responsible for moving a propeller plane forward is the primary lift force in the case of y4 copter. Propeller design also factors in as the blade turns outward from the center. This results in a change of the angle of attack (AOA) to its optimum angle at the tips of each propeller blade.

The other force to be considered is the lateral forces being induced. Normally, there is no drag forces present in hover because of the dual blade propeller moving at high speeds. The torque generated from the motor results in a rotational force, causing the entire craft to spin, hence the second blade (called the anti-torque blade) to counteract the forces of the torque. For the y4 copter, the torques of the counter rotating blades cancels each other out thus, preventing the system from drift.

Another design consideration is the Magnus effect which assumes a cylinder of infinite length which rotates. Air has a tendency to induce a lift if the rotation of the cylinder is moving relatively upwards. In this case, the force is deemed negligible in still air at hover. But, in case of turbulence, it is possible for this force to become imbalanced generating a drift force. Initial takeoff circumstances must also be taken into account. At start-up, the capability of a slow increase in the propellers' speed to avoid a fast take-off is anticipated [3]. Fast take-offs has long term stress and shearing effects on the internal bolts of the craft. Grounding effects is also a leading factor in faster take-off as more air being pushed back into the airflow of the blades leading to an initial cyclonic effect surrounding the blades and creating further stress on the frame and the propellers.

\subsection{Forward Motion}

Forward motion is an issue that involves propellers. Generally, blades on the main rotor can tilt in a way to create the condition for forward flight. In the design of y4 copter, the propeller blades remain relatively static to the center piece. To create forward flight, the whole copter is to be tilted by reducing the lift speed of at least one motor. Timing is crucial for sustaining altitude. The optimum angle the copter has to maintain to sustain altitude, with a maximum forward thrust, is normally no more than 10-15 degrees. Placement of the sensors for feedback to the processor is also to be considered. For optimal response, the accelerometer handles lateral and vertical 
feedback where a gyroscope handles all rotation feedback. The orientation of each device is considered for controlling flight.

For the designed fixed pitch y4 copter, the AOA is changed for maneuvering by tilting the whole body without any AOA for lift. Forward flight denotes a change in angle of the copter therefore a change in the AOA causing a drop in the altitude. To compensate for this, the lift used in hovering is set as the forward thrust. This new lift is the upward vertical force, which is equal to the lift force in hover before forward motion to sustain altitude. The new equations generated from forward thrust are given as follows:

$$
\text { Lift equation: } \mathrm{LF}=\mathrm{L}_{\text {hover }} \times \cos \zeta
$$

$$
\text { Forward Thrust equation: } \mathrm{TF}=\mathrm{L}_{\mathrm{hover}} \times \sin \zeta
$$

Here $\mathrm{L}_{\text {hover }}$ denotes the lift in hover before forward motion which can be determined by centrifugal force, and $\zeta$ is the forward tilt angle.

Once the copter is in a constant forward motion, the net forces are considered to be zero. Then the velocity of the copter can be considered in a linear perspective. This can be said if it is assumed that the plane of the copter's propellers is a solid and the mass is centered. This paves the way for a steady-state velocity to be established. This velocity can be determined by the linear momentum equation. Next, turning is also an important issue, since forward flight attitude requires more power. Then the revised equations from above are as follows:

$$
\text { Lift equation: } \quad \mathrm{LF}=\mathrm{L}_{\text {hover }} \times \cos \zeta \times \cos \theta
$$

Thrust equation: $\mathrm{TFT}=\mathrm{L}_{\text {hover }} \times \sin \zeta \times \sin \theta$

Here, theta $(\theta)$ is the pitch angle to be determined. Again, the range of $\theta$ must be kept between 515 degrees.

\subsection{System Modeling}

For modeling the system, a few necessary assumptions are made. The body of the copter is assumed to be rigid considering the effect of bending rotors negligible. Controllers are assumed to be fast enough to neglect dynamics of the motor and changing angular rate. Gyro effects due to the angular momentum of the rotors are also ignored. The model of the structure is illustrated in Fig. 3. The input vector is defined as $\mathbf{u}=\left[\mathrm{u}_{\Phi} \mathrm{u}_{\theta} \mathrm{u}_{\psi} \mathrm{u}_{\text {thrust }}\right]$ containing commands for roll, pitch, yaw and thrust. The input serves as references to the on-board heading hold controller holding the desired angular velocity in the body frame $\left({ }^{b} \boldsymbol{\omega}\right)$. The force generated by the rotors $\left(\mathbf{F}_{\text {liff }}\right)$ is derived using the information about the on-board controller. Then an expression of the acceleration in body frame $\left({ }^{\mathrm{b}} \mathbf{v}\right)$ is derived using the force generated by the lift. 


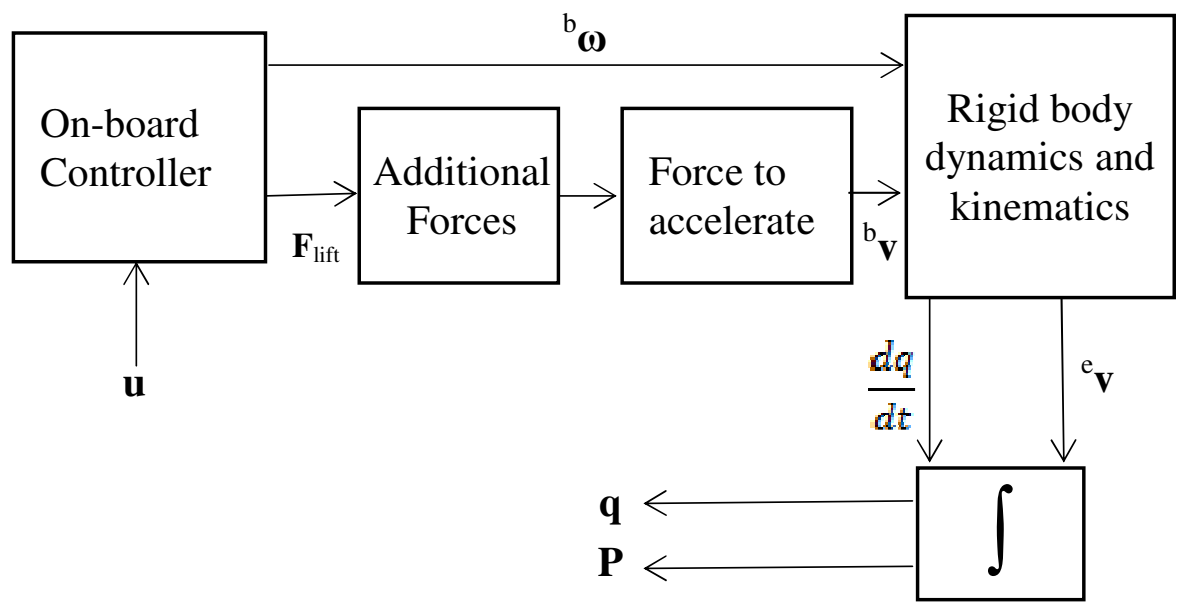

Figure 3. Model structure

The model completes by including the final integrations and rotations into the earth fixed frame deriving the time derivative of the orientation (q) and position (P) in the earth frame. Then the model is linearized and converted to state-space form.

\subsubsection{Mathematical Modeling}

\begin{tabular}{|l|l|}
\hline \multicolumn{2}{|c|}{ Notations } \\
\hline$f 1, f 2, f 3, f 4$ - individual thrust of four rotors & $p$-roll rate \\
$F_{\text {rotor }}$ - total thrust of the quad copter & $q$ - pitch rate \\
$\varphi$ - roll angle & $r$-yaw rate \\
$\theta$ - pitch angle & $\begin{array}{l}u \text { - longitudinal speed in body-fixed } \\
\text { frame } \\
\psi \text { - yaw angle } \\
\begin{array}{l}x, y, z \text { - longitudinal, lateral and vertical coordinate in } \\
\text { Earth-fixed frame respectively }\end{array}\end{array}$ \\
& $\begin{array}{l}w \text { - lateral speed in body fixed frame } \\
\end{array}$ \\
\hline
\end{tabular}

Table 1. Notation followed

Assuming a symmetric structure and the origin of body frame coincided with the center of mass, the linear and angular velocities are given as,

$$
V_{B}=\left[\begin{array}{l}
v_{x, B} \\
v_{y, B} \\
v_{z, B}
\end{array}\right], \quad v=\left[\begin{array}{l}
p \\
q \\
r
\end{array}\right]
$$

And the rotation matrix to convert from body frame to inertial frame is, 
International Journal of Information Technology, Control and Automation (IJITCA) Vol.3, No.2, April 2013

$$
R=\left[\begin{array}{ccc}
\cos \varphi \cos \theta & \cos \psi \sin \theta \sin \varphi-\sin \psi \cos \varphi & \cos \psi \sin \theta \cos \varphi+\sin \psi \sin \varphi \\
\sin \varphi \cos \theta & \sin \psi \sin \theta \sin \varphi+\cos \psi \cos \varphi & \sin \psi \sin \theta \cos \varphi-\cos \psi \sin \varphi \\
-\sin \theta & \cos \theta \sin \varphi & \cos \theta \cos \varphi
\end{array}\right]
$$

Due to its orthogonal nature, the transpose matrix of $\mathrm{R}$ is the rotation matrix to convert from inertial frame to body frame. To transform the velocities from inertial frame to body frame and vice versa, the following set of equations is used.

$$
\begin{aligned}
& \dot{\eta}=W_{\eta}^{-1} v \\
& {\left[\begin{array}{c}
\dot{\varphi} \\
\dot{\theta} \\
\dot{\psi}
\end{array}\right]=\left[\begin{array}{ccc}
1 & \sin \varphi T_{\theta} & \cos \varphi T_{\theta} \\
0 & \cos \varphi & -\sin \varphi \\
0 & \sin \varphi / \cos \theta & \cos \varphi / \cos \theta
\end{array}\right]\left[\begin{array}{l}
p \\
q \\
r
\end{array}\right]} \\
& \dot{v}=W_{\eta} \eta \\
& {\left[\begin{array}{l}
p \\
q \\
r
\end{array}\right]=\left[\begin{array}{ccc}
1 & 0 & -\sin \theta \\
0 & \cos \varphi & \cos \theta \sin \varphi \\
0 & -\sin \varphi & \cos \theta \cos \varphi
\end{array}\right]\left[\begin{array}{c}
\dot{\varphi} \\
\dot{\theta} \\
\dot{\psi}
\end{array}\right]}
\end{aligned}
$$

The thrust force, $T$, is the combined force given by all four rotors as all of them provide thrust along the same direction. Torque, $\tau_{B}$ created by the rotors, consists of $\tau_{\varphi}, \tau_{\theta}$ and $\tau_{\psi}$ in the direction of the corresponding body frame angles. The working sets of equations are given by:

$$
\begin{gathered}
T=\sum_{i=1}^{4} f_{i}=k \sum_{i=1}^{4} \omega_{i}^{2}, \boldsymbol{T}^{B}=\left[\begin{array}{c}
0 \\
0 \\
T
\end{array}\right] \\
\tau^{B}=\left[\begin{array}{c}
\tau_{\varphi} \\
\tau_{\theta} \\
\tau_{\psi}
\end{array}\right]=\left[\begin{array}{c}
l k\left(-\omega_{2}^{2}+\omega_{4}^{2}\right) \\
l k\left(-\omega_{1}^{2}+\omega_{3}^{2}\right) \\
\sum_{i=1}^{4} \tau_{M_{i}}
\end{array}\right]
\end{gathered}
$$

In this case, one set of state equations that derives from the linear acceleration equations are,

$$
\begin{aligned}
& x^{\prime \prime}=\psi^{\prime} y^{\prime}-\theta^{\prime} z^{\prime}-g \sin \theta \\
& y^{\prime \prime}=\varphi^{\prime} z^{\prime}-\psi^{\prime} x^{\prime}+g \cos \theta \sin \varphi \\
& z^{\prime \prime}=\theta^{\prime} \psi^{\prime}-\varphi^{\prime} y^{\prime}+g \cos \theta \cos \varphi-\frac{b}{m}\left(\Omega_{1}^{2}+\Omega_{2}^{2}+\Omega_{3}^{2}+\Omega_{4}^{2}\right)
\end{aligned}
$$

And the other set that derives from the angular acceleration equations are,

$$
\begin{gathered}
\varphi^{\prime \prime}=\frac{b l}{I_{x}}\left(\Omega_{2}^{2}-\Omega_{4}^{2}\right)-\theta^{\prime} \psi^{\prime} \frac{I_{z}-I_{y}}{I_{x}} \\
\theta^{\prime \prime}=\frac{b l}{I_{y}}\left(\Omega_{1}^{2}-\Omega_{3}^{2}\right)-\varphi^{\prime} \psi^{\prime} \frac{I_{x}-I_{z}}{I_{y}}
\end{gathered}
$$




$$
\psi^{\prime \prime}=\frac{d}{I_{z}}\left(\Omega_{2}^{2}+\Omega_{4}^{2}-\Omega_{1}^{2}-\Omega_{3}^{2}\right)
$$

Here $\Omega_{i}$ denotes the angular velocities of the individual rotors which control both the thrust and the torques. $I_{x}, I_{y}$ and $I_{z}$ are the moment of inertia for the whole structure which must be determined experimentally. $b$ is the thrust coefficient, $d$ is the drag coefficient of rotating propeller and $l$ is the distance of each rotor from the center of gravity of the structure assuming $l$ remains same for all rotors, and value of these also must be determined experimentally.

\subsection{Flight Principle}

The designed y4 copter is equipped with four motors with each a rotor attached. Due to the spinning of the rotor, lift is generated. When aligned with the horizontal plane and the sum of the lift generated $\left(\mathrm{F}_{\text {lift }}\right)$ becomes equal to the gravitational force, the copter hovers. If $\mathrm{F}_{\text {lift }}$ is increased, it climbs and if $F_{\text {lift }}$ is decreased it descents.

When the copter is tilted, the direction of $F_{\text {lift }}$ is no longer aligned with the earth frame z-axis but the force can be resolved in a z-axis component and a horizontal component which is depicted in Fig. 4. The horizontal component results in acceleration in the horizontal plane.

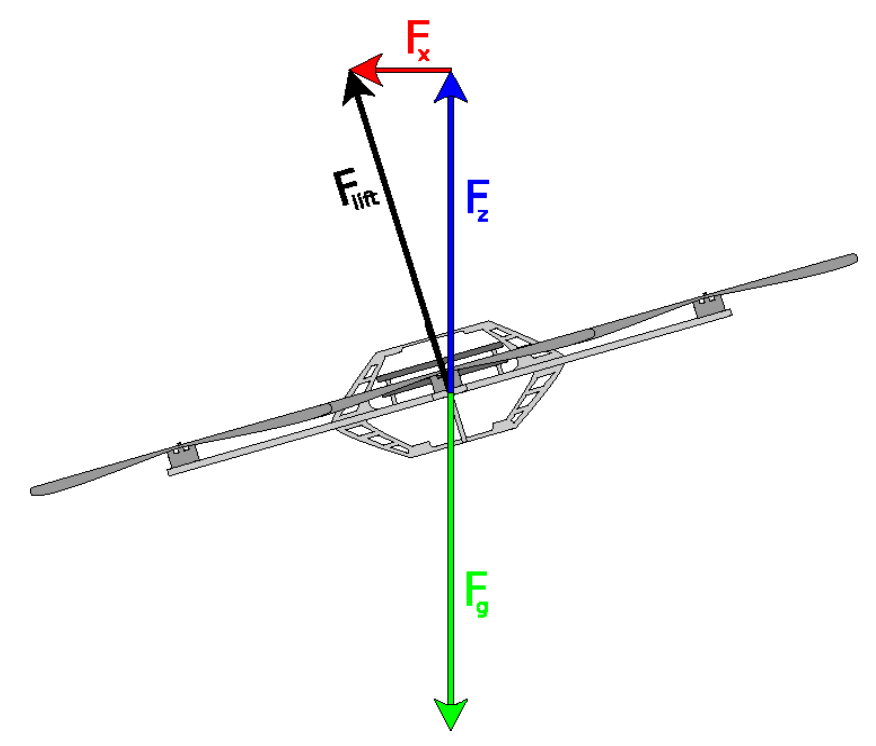

Figure 4. Resolving the lift force into horizontal and vertical components

\subsubsection{Flight Control Principle}

The rotors spin in opposite direction in pairs to prevent the y4 copter from spinning around the zaxis resulting from an effect of the counter torque generated by the spinning rotors. When the rotors spin in opposite direction, the counter torque is equalized if all rotors rotate with equal speed. Tilting of the y4 copter can be done by altering the speed of the rotors.

As demonstrated in Fig. 5, roll refers to whether the body is upside-down or not i.e., orientation of the body within the yz plane, or rotating around the $\mathrm{x}$ axis. Pitch infers whether the body is tilted i.e., its orientation within the $\mathrm{xz}$ plane, or rotating around the $\mathrm{y}$ axis. Yaw refers to the 
direction in which the body is facing i.e., its orientation within the xy plane, or rotating around the $\mathrm{z}$ axis.

To achieve forward pitch, speed of the front two rotors must be decreased and that of the rear two rotors must be increased proportionally keeping the net vertical thrust same to maintain altitude. To perform roll, speed of any of the front two rotors must be decreased and that of the other must increase. For anticlockwise yaw, the upper one of the rear rotors must be in higher speed than the lower one. Table 2 demonstrates the flight control principle.

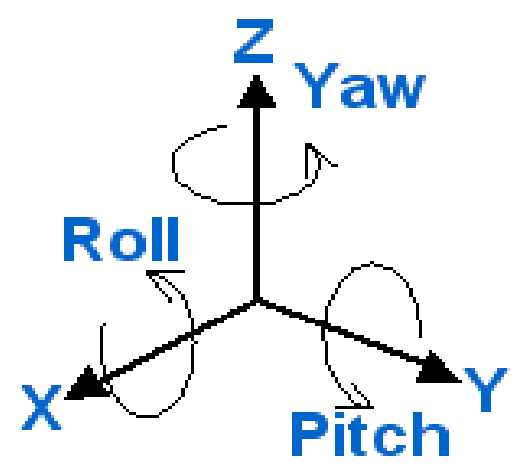

Figure 5. Visual representation of roll, pitch and yaw

\begin{tabular}{|l|l|}
\hline Action & Result \\
\hline Thrust increase & $1,2,3,4$ speed up \\
\hline Thrust decrease & $1,2,3,4$ speed down \\
\hline Forward pitch & 1,2 speed down \& 3,4 speed up \\
\hline Backward pitch & 1,2 speed up \& 3,4 speed down \\
\hline Positive roll & 1 speed up 2 speed down \\
\hline Negative roll & 1 speed down 2 speed up \\
\hline Positive yaw & 3 speed down 4 speed up \\
\hline Negative yaw & 3 speed up 4 speed down \\
\hline
\end{tabular}

Table 2. Necessary Rotor $(1,2,3,4)$ actions for desired flight management

\section{SYSTEM DEVELOPMENT}

The building blocks of the developed system are illustrated in Fig. 6 . 


\section{BASE STATION}

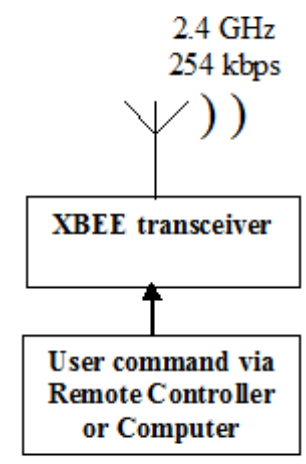

ON BOARD SYSTEM

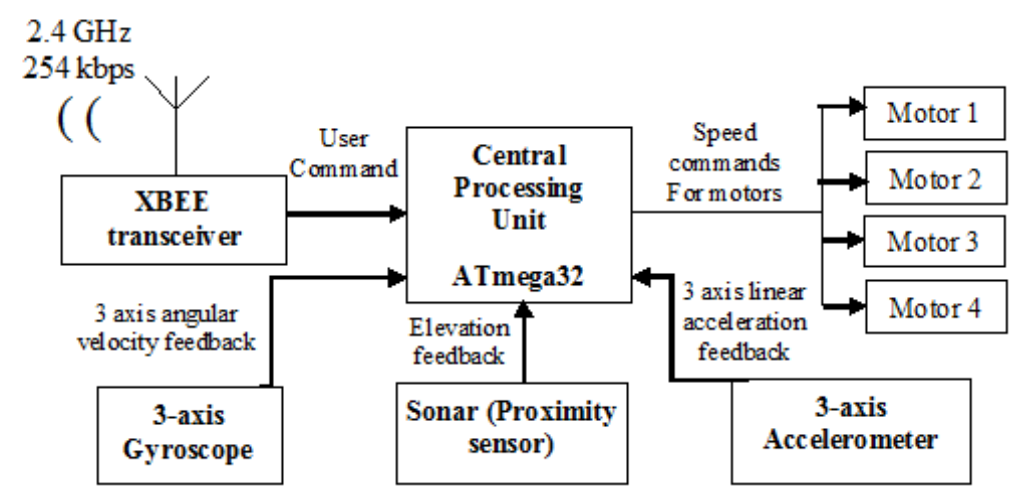

Figure 6. Block diagram of the overall system

\subsection{Inertial Measurement Unit (IMU)}

The combination of accelerometers and gyroscopes is a common approach to measure and stabilize the flight. Inertial measurement unit (IMU) is the combination of different sensors operating together to establish an accurate orientation measurement relative to the ground. IMU's are available that vary in the degree of complexity according to the number of axes that are measured by employing different sensors. For instance, 3 degrees of freedom IMU combo board can measure up to 3 axes with one type of sensor. On the other hand, 9 degrees of freedom IMU measures each axis with three different sensor types. The third sensor can be a magnetometer. To monitor the tilt of the $\mathrm{y} 4$ copter, the rotation about 2 axes $(\mathrm{X}$ and $\mathrm{Y})$ or 2 degrees of freedom are to be measured at minimum. Gyroscopes, accelerometers or a combination of both can be used to measure these axes.

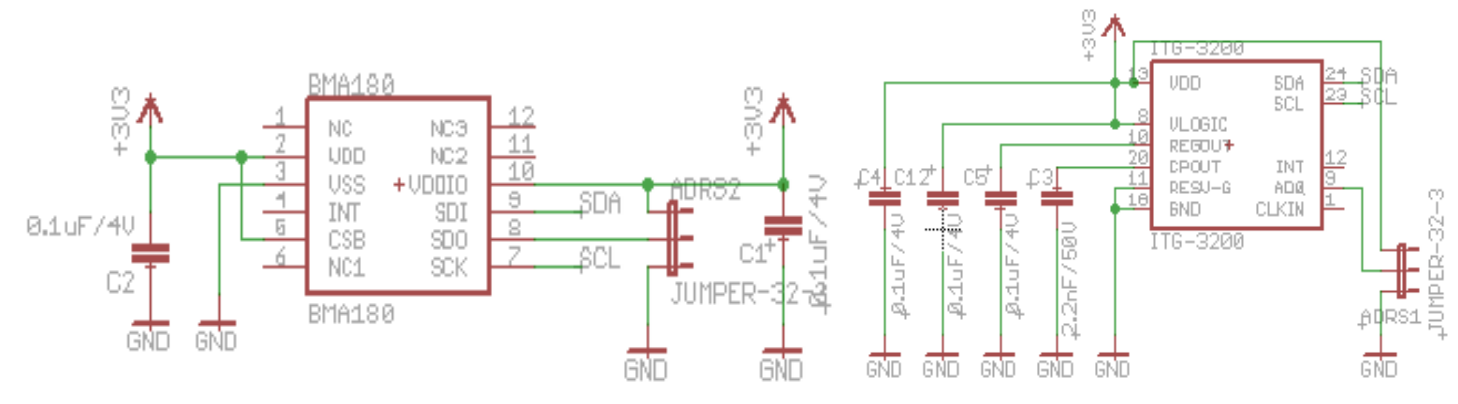

(a)

(b)

Figure 7. Wiring schematic and power regulation of (a) BMA 180 accelerometer and (b) ITG 3200 gyroscope

A combination of accelerometers and gyroscopes measuring the same axis may appear redundant but, the methods of the two sensors differ and thus a more complete picture can be conveyed to the microcontroller for processing. For these reasons a 6 degrees of freedom IMU is integrated into the design of the 44 copter. This entails a triple axis accelerometer and a triple axis gyroscope and with this configuration each of the 3 critical axes are monitored by 2 different 
sensor types. Triple Axis Digital-Output Gyro ITG-3200 Breakout [4] \& Triple Axis Accelerometer Breakout BMA180 [5] are used as gyroscope and accelerometer respectively. Their wiring schematics along with power regulation are shown in Fig. 7.

\subsection{Calculation of Euler Angle from Quaternion}

There is an inherent problem in IMU which is referred as gimbal lock. It is the loss of one degree of freedom in a three-dimensional space that occurs when the axes of two of the three gimbals are driven into a parallel configuration, "locking" the system into rotation in a degenerate twodimensional space. A case of a level sensing platform on an aircraft flying due north can be considered as an example. It has three gimbal axes which are mutually perpendicular (i.e., roll, pitch and yaw angles each zero). If the aircraft pitches up 90 degrees, the aircraft and platform's Yaw axis gimbal becomes parallel to the Roll axis gimbal. Then the changes about yaw cannot be compensated. One and most efficient solution is the use of quaternions. A quaternion describes a rotation in one single move while the Euler angles (Fig. 8) are made of three successive rotations. So, there is no gimbal lock problem.

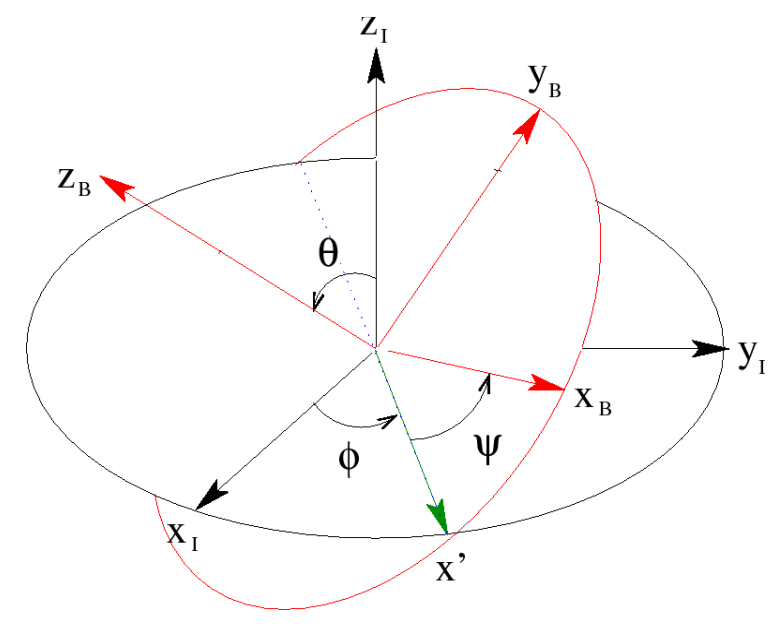

Figure 8. Euler angles

A unit quaternion: $\quad \mathrm{q}=\left[\mathrm{q}_{0} \mathrm{q}_{1} \mathrm{q}_{2} \mathrm{q}_{3}\right]^{\mathrm{T}}$

$$
|q|^{2}=q_{0}^{2}+q_{1}^{2}+q_{2}^{2}+q_{3}^{2}=1
$$

A quaternion can be associated with a rotation around an axis by the following expression.

$$
\begin{aligned}
& \mathrm{q}_{0}=\cos (\alpha / 2) \\
& \mathrm{q}_{1}=\sin (\alpha / 2) \cos \left(\beta_{\mathrm{x}}\right) \\
& \mathrm{q}_{2}=\sin (\alpha / 2) \cos \left(\beta_{\mathrm{y}}\right) \\
& \mathrm{q}_{3}=\sin (\alpha / 2) \cos \left(\beta_{\mathrm{z}}\right)
\end{aligned}
$$


Where $\alpha$ is the value in radians of the angle of rotation and $\cos \left(\beta_{x}\right), \cos \left(\beta_{y}\right)$ and $\cos \left(\beta_{z}\right)$ are the direction cosines locating the axis of rotation according to Euler's Theorem. The orthogonal matrix after multiplying a column vector corresponding to a clockwise rotation by the unit quaternion $q=q_{0}+i q_{1}+j q_{2}+k q_{3}$ is given by the following inhomogeneous expression:

$$
\left[\begin{array}{lll}
1-2\left(q_{2}^{2}+q_{3}^{2}\right) & 2\left(q_{1} q_{2}-q_{0} q_{3}\right) & 2\left(q_{0} q_{2}+q_{1} q_{3}\right) \\
2\left(q_{1} q_{2}+q_{0} q_{3}\right) & 1-2\left(q_{1}^{2}+q_{3}^{2}\right) & 2\left(q_{2} q_{3}-q_{0} q_{1}\right) \\
2\left(q_{1} q_{3}-q_{0} q_{2}\right) & 2\left(q_{0} q_{1}+q_{2} q_{3}\right) & 1-2\left(q_{1}^{2}+q_{2}^{2}\right)
\end{array}\right]
$$

Which, equivalently, can be expressed by the homogeneous expression:

$$
\left[\begin{array}{ccc}
q_{0}^{2}+q_{1}^{2}-q_{2}^{2}-q_{3}^{2} & 2\left(q_{1} q_{2}-q_{0} q_{3}\right) & 2\left(q_{0} q_{2}+q_{1} q_{3}\right) \\
2\left(q_{1} q_{2}+q_{0} q_{3}\right) & q_{0}^{2}-q_{1}^{2}+q_{2}^{2}-q_{3}^{2} & 2\left(q_{2} q_{3}-q_{0} q_{1}\right) \\
2\left(q_{1} q_{3}-q_{0} q_{2}\right) & 2\left(q_{0} q_{1}+q_{2} q_{3}\right) & q_{0}^{2}-q_{1}^{2}-q_{2}^{2}+q_{3}^{2}
\end{array}\right]
$$

If $q_{0}+i q_{1}+j q_{2}+k q_{3}$ is not a unit quaternion, then the homogeneous form still remains to be a scalar multiple of a rotation matrix. On the other hand the inhomogeneous form is in general no longer an orthogonal matrix. Due to this fact, to avoid the distortion, the homogeneous form needs to be preferred in numerical work

The orthogonal matrix corresponding to a clockwise rotation with Euler angles $\varphi, \theta, \psi$, with x-y$\mathrm{z}$ convention, is given by:

$$
\left[\begin{array}{ccc}
\cos \theta \cos \psi & -\cos \emptyset \sin \psi+\sin \emptyset \sin \theta \cos \psi & \sin \emptyset \sin \psi+\cos \emptyset \sin \theta \cos \psi \\
\cos \theta \sin \psi & \cos \emptyset \cos \psi+\sin \emptyset \sin \theta \sin \psi & -\sin \emptyset \cos \psi+\cos \emptyset \sin \theta \sin \psi \\
-\sin \theta & \sin \emptyset \cos \theta & \cos \emptyset \cos \theta
\end{array}\right]
$$

Combining the quaternion representations of the Euler rotations we obtain,

$$
\begin{aligned}
\mathrm{q}=\mathrm{Rz}(\psi) \operatorname{Ry}(\theta) \operatorname{Rx}(\phi)=[\cos (\psi / 2)+\mathbf{k} \sin (\psi / 2)][\cos (\theta / 2)+\mathbf{j} \sin (\theta / 2)][\cos (\phi / 2)+\mathbf{i} \sin (\phi / 2)] \\
\mathrm{q}=\left[\begin{array}{l}
\cos (\phi / 2) \cos (\theta / 2) \cos (\psi / 2)+\sin (\phi / 2) \sin (\theta / 2) \sin (\psi / 2) \\
\sin (\phi / 2) \cos (\theta / 2) \cos (\psi / 2)-\cos (\phi / 2) \sin (\theta / 2) \sin (\psi / 2) \\
\cos (\phi / 2) \sin (\theta / 2) \cos (\psi / 2)+\sin (\phi / 2) \cos (\theta / 2) \sin (\psi / 2) \\
\cos (\phi / 2) \cos (\theta / 2) \sin (\psi / 2)-\sin (\phi / 2) \sin (\theta / 2) \cos (\psi / 2)
\end{array}\right]
\end{aligned}
$$

For Euler angles, the relation is:

$$
\left[\begin{array}{l}
\phi \\
\theta \\
\psi
\end{array}\right]=\left[\begin{array}{c}
\arctan \frac{2\left(q_{0} q_{1}+q_{2} q_{3}\right)}{1-2\left(q_{1}^{2}+q_{2}^{2}\right)} \\
\arcsin \left(2\left(q_{0} q_{2}-q_{3} q_{1}\right)\right) \\
\arctan \frac{2\left(q_{0} q_{3}+q_{1} q_{2}\right)}{1-2\left(q_{2}^{2}+q_{3}^{2}\right)}
\end{array}\right]
$$

"arctan" and "arcsin" have a result between $-\pi / 2$ and $\pi / 2$. With three rotations between $-\pi / 2$ and $\pi / 2$, all possible orientations cannot be obtained. arctan needs to be replaced by atan 2 to generate all the orientations. 


$$
\left[\begin{array}{l}
\phi \\
\theta \\
\psi
\end{array}\right]=\left[\begin{array}{c}
\operatorname{atan} 2\left(2\left(q_{0} q_{1}+q_{2} q_{3}\right), 1-2\left(q_{1}^{2}+q_{2}^{2}\right)\right) \\
\arcsin \left(2\left(q_{0} q_{2}-q_{3} q_{1}\right)\right) \\
\operatorname{atan} 2\left(2\left(q_{0} q_{3}+q_{1} q_{2}\right), 1-2\left(q_{2}^{2}+q_{3}^{2}\right)\right)
\end{array}\right]
$$

[6]- [7] have been consulted for selection and operation of quaternion in IMU.

\subsection{Automated Elevation}

LV-MaxSonar-EZ0 ${ }^{\mathrm{TM}}$ High Performance Sonar Range Finder [8] is used for measuring the elevation of the y4 copter. LV-MaxSonar®- ${ }^{\circledR} Z 0^{\mathrm{TM}}$ requires $2.5 \mathrm{~V}-5 \mathrm{~V}$ supply. It provides very short to long-range detection and ranging. It is capable of detecting objects from 0 -inches to 254inches (6.45-meters) besides providing sonar range information from 6-inches out to 254-inches with 1-inch resolution.

Fig. 9 shows the low-pass filter used before giving the power supply according to the suggestion of Maxbotix. The LV-MaxSonar-EZ0 ${ }^{\mathrm{TM}}$ is the most sensitive of the MaxSonar product line, yielding a controlled wide beam with very high sensitivity [8]. The LV-MaxSonar-EZ0TM supports RS-232protocol and also offers pulse width representation and analog output.

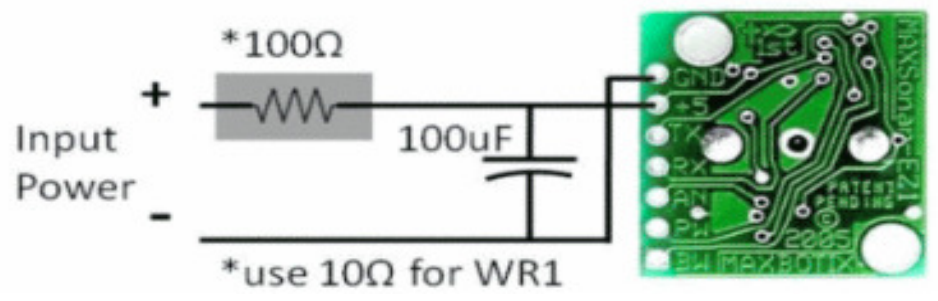

Figure 9. MaxBotix recommended low-pass filter [8].

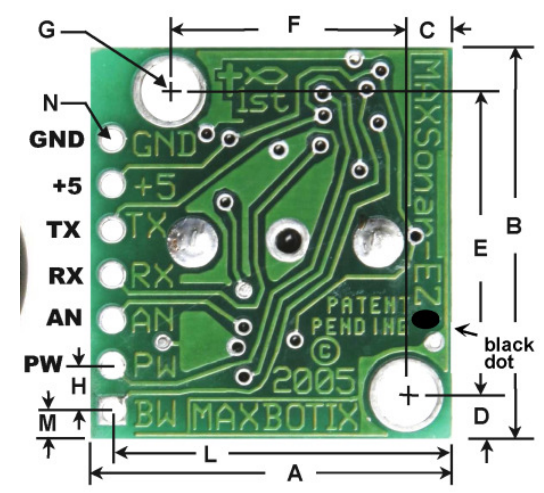

Figure 10. Connection terminals of LV-MaxSonar-EZ0 ${ }^{\mathrm{TM}}$ 

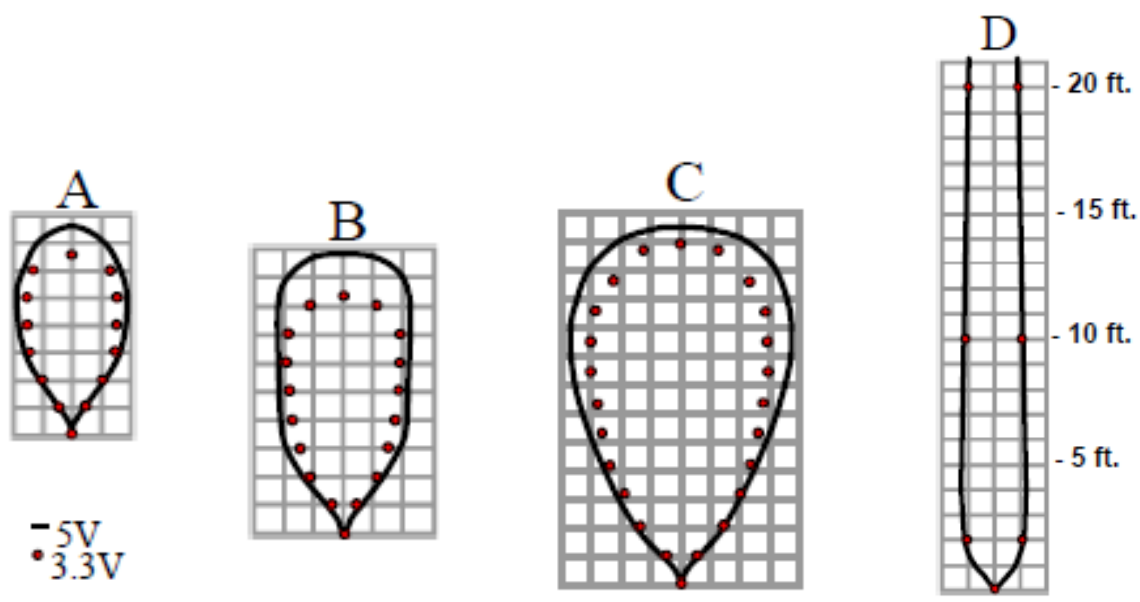

Figure 11. LV-MaxSonar®-EZ0 ${ }^{\mathrm{TM}}$ beam detection pattern diagram for (a) 0.25-inch diameter

dowel (narrow beam for close small objects), (b) 1-inch diameter dowel (the long narrow detection pattern), (c) 3.25-inch diameter rod (the long controlled detection pattern), (d) 11-inch wide board moved left to right with the board parallel to the front sensor face keeping the sensor stationary [8]

Fig. 10 shows the connection terminals of LV-MaxSonar-EZ0 ${ }^{\mathrm{TM}}$. When the BW is open or kept low, the TX output delivers asynchronous serial data with an RS232 format, except voltages are $0-\mathrm{Vcc}$. The output is an ASCII capital "R", followed by three ASCII character digits representing the range in inches up to a maximum of 255, followed by a carriage return (ASCII 13). The baud rate for communication is 9600,8 bits, no parity, with one stop bit. For the prototype, RS-232 protocol is used using MAX 232 converter [9] for getting standard voltage level. Sample results for measured beam patterns are shown in Fig. 11 on a 12-inch grid.

\subsection{Control System Design}

Proportional Integral Derivative (PID) control system is employed for controlling roll, pitch, and yaw and also for controlling elevation. In PID controller, the effects of each controller is added in order to obtain fast and stable response. The stationary error of the $\mathrm{P}$ controller is offset by the integral term and the overshoot of the $\mathrm{P}$ controller is offset by the differential term and thus makes the response faster. Hence for the y4 copter, the PID controller is chosen.

On the y4 copter, a 3-axis accelerometer and a 3-axis gyroscope are the sensors used in order to provide the linear control system with an output signal. The IMU system is tested in order to get the reference signal that conveys the linear control system that the y4 copter is steady and stable. The accelerometer is used to determine pitch and roll while the gyroscope is used as a heading indicator or yaw sensor. The accelerometer and gyroscope have errors in their reading that requires to be filtered before using them as sensors for the linear control system. A gyroscope has drift that makes it accumulate error over time rendering gyroscope to perform great at sensing for a short period of time; but poor at sensing for a long period of time. While the accelerometer is great at sensing for a long period of time, it is susceptible to noise. In order to get a good signal out these sensors their output has to be combined. The way that they are combined is through the 
use of an averaging system. Using a filter the output of the IMU system can then be used in PID controller. Proximity sensor is used to determine the current elevation to be given as the feedback signal for the PID controller used for automatic elevation and landing.

The PID controller shown in Fig. 12 is used to correct deviation from the desired output. The variables that are controlled with the linear control system are the roll, pitch, yaw and elevation of the y4 copter. The current roll, pitch and yaw are sensed by the y4 copter's accelerometer and gyroscope. This signal passes through a filter, which is implemented on an 8-bit microcontroller AVR ATmega 32 [10]. This signal then passes through the PID controller, also implemented on the same microcontroller. The PID then calculates the amount of thrust needed to get the system to the desired output. There are three PID controllers for flight stabilization: one for each of the roll, pitch and yaw. There is also a PID controller for automated elevation which takes the output of the proximity sensor as its feedback. The microcontroller cycles through each PID at a set interval until the desired output is reached. The ideal parallel form of the PID controller equation is continuous which is not suitable for implementation on an 8-bit microcontroller. The equation is to be transformed into a discrete equation.

The ideal parallel form equation is given by

$$
\mathrm{U}(\mathrm{t})=\mathrm{MV}(\mathrm{t})=\mathrm{K}_{\mathrm{p}} \mathrm{e}(\mathrm{t})+\mathrm{K}_{\mathrm{i}} \int_{0}^{t} e(\tau) d \tau+\mathrm{K}_{\mathrm{d}} \frac{d e(t)}{d t}
$$

Where, $K_{p}$ is the Proportional gain; $K_{i}$ denotes the Integral gain; $K_{d}$ is the Derivative gain; e denotes the Error $=\mathrm{SP}-\mathrm{PV}$ and $\mathrm{t}$ is the Time or instantaneous time. In order to discretize the PID controller equation, the integral term and the derivative term has to be expressed in discrete form. To discretize the derivative term, a backward finite difference form is used. For the integral term, a summation of all previous error is used for calculation. The error signal is a discrete function where each error is sampled and stored in an array. The derivative term calculates the difference between two consecutive error samples and divides it by the sampling period. On the other hand, the integral term does a summation of the array and multiplies it by the sampling frequency. The discrete equation can be written as:

$$
\begin{aligned}
& \mathrm{x}(\mathrm{n})=\mathrm{K}_{\mathrm{p}} \mathrm{e}(\mathrm{n})+\mathrm{K}_{\mathrm{i}} \Sigma \mathrm{e}(\mathrm{k})+\mathrm{K}_{\mathrm{d}}[\mathrm{e}(\mathrm{n})-\mathrm{e}(\mathrm{n}-1)] \\
& \mathrm{K}_{\mathrm{i}} \text { term: } \quad \mathrm{K}_{\mathrm{i}}=\mathrm{K}_{\mathrm{p}} \mathrm{T} / \mathrm{T}_{\mathrm{i}} \\
& \mathrm{K}_{\mathrm{d}} \text { term: } \mathrm{Kd}=\mathrm{K}_{\mathrm{p}} \mathrm{T}_{\mathrm{d}} / \mathrm{T}
\end{aligned}
$$

Where, $\mathrm{T}$ is the sampling period and Ti denotes the integral time constant.

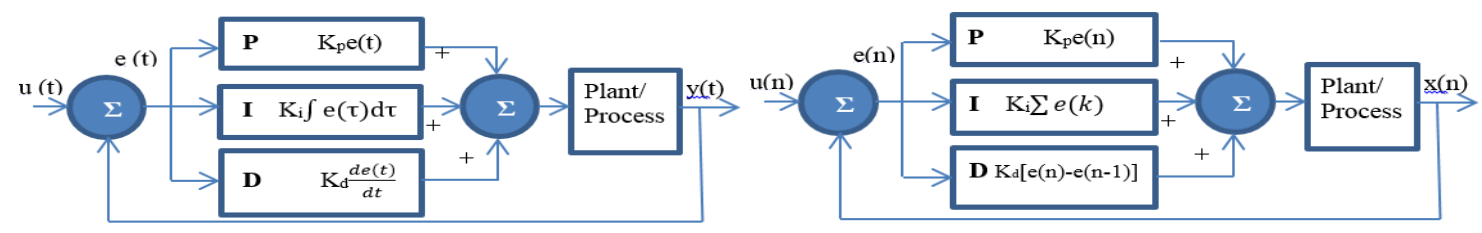

(a)

(b)

Figure 12. PID Controller: (a) for continuous time form and (b) in digitally implementable form 
The values of $\mathrm{K}_{\mathrm{p}}, \mathrm{K}_{\mathrm{d}}$ and $\mathrm{K}_{\mathrm{i}}$ for each PID controller can be obtained by trial and error which is actually the method that has been followed earlier [1]. To obtain greater flexibility, ZieglerNichols method [11] is used. The Ziegler-Nichols method begins with setting the $K_{i}$ and $K_{d}$ to 0 . Then $K_{p}$ is raised until the output oscillates. The gain at which the oscillation happens, is called the ultimate gain or gain margin, $\mathrm{K}_{\mathrm{u}}$. The period of the oscillation is termed as $\mathrm{P}_{\mathrm{u}}$. After that simple equations are used in order to find $T_{i}, T_{d}, K_{i}$, and $K_{d}[12]$ as shown in Table 3.

\begin{tabular}{|c|c|c|c|}
\hline \multicolumn{4}{|c|}{ Ziegler-Nichols method } \\
\hline Control Type & $\boldsymbol{K}_{p}$ & $\boldsymbol{K}_{\boldsymbol{i}}$ & $\boldsymbol{K}_{\boldsymbol{d}}$ \\
\hline $\boldsymbol{P}$ & $0.50 K_{u}$ & - & - \\
\hline $\boldsymbol{P I}$ & $0.45 K_{u}$ & $1.2 K_{p} / P_{u}$ & - \\
\hline $\boldsymbol{P I D}$ & $0.60 K_{u}$ & $2 K_{p} / P_{u}$ & $K_{p} P_{u} / 8$ \\
\hline
\end{tabular}

Table 3. Gains for different types of control systems using Ziegler-Nichols method

According to Ziegler-Nichols rule, the system has a transfer function of the form given below:

$$
\frac{K e^{-s T}}{(s+a)}
$$

The model matches the system response at two frequencies: 0 and at the stability limit. Everything else in between is more or less made up. The model parameters are:

$$
\text { Model's 'a' term: } a=\sqrt{K^{2} K u^{2}-4 \pi F u^{2}}
$$

Where, Fu is the oscillation frequency.

Phase shift: $\phi=-\tan ^{-1}\left(2 \pi \frac{F u}{a}\right)$

Delay term: $\mathrm{T}=\frac{(-\Pi-\phi)}{2 \Pi F u}$

The 8-bit microcontroller limits how well the PID controller can be implemented. It has a low resolution that has to be taken in to account in calculating the PID controller term. Lastly it doesn't have floating point capability which means the PID controller equation is to be implemented with integer math. 


\subsection{Wireless Communication between the Base and the Copter}

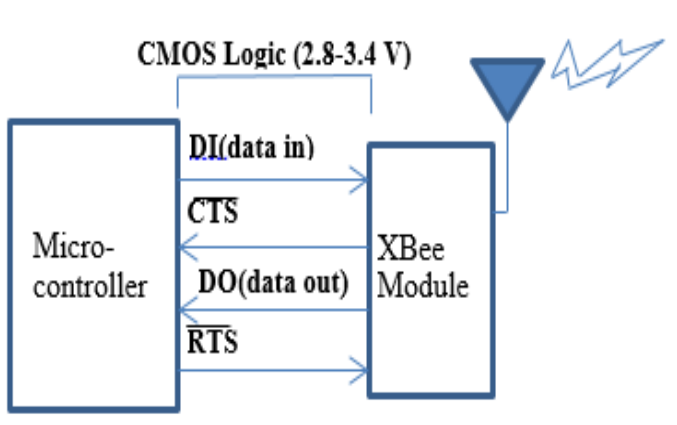

(a)

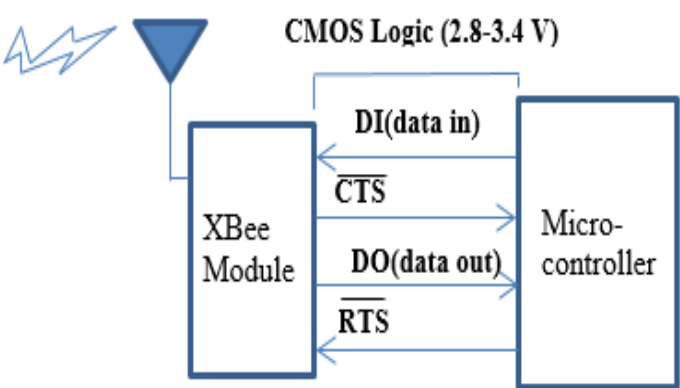

(b)

(b)

Figure 13. Serial communication between XBee and microcontroller: (a) transmission side, (b) receiving side [13]

The 2.4 GHz XBee XBP24-ACI-001 transceiver module from Digi is used for wireless communication between the on board controller and the base station which takes the 802.15.4 stack which is the basis for Zigbee and wrap it into a simple to use serial command set. This module allows a very reliable and simple communication between microcontrollers, computers and systems. Point to point and multi-point networks are supported too. Atmel AVR ATmega 32 [10] microcontroller is used to communicate serially with XBee. ATmega 32 has built-in Universal Asynchronous serial Receiver Transmitter (UART) protocol. The baud rate used is 9600, 8 bits data, no parity, with one stop bit. Serial communication established between XBee module and microcontroller is demonstrated in Fig. 13.

XBee Pro 60mW Chip Antenna having 1 mile range of communication [13] is used for XBee communication. The necessary configuration is done using AT commands. The syntax for sending AT commands is shown in Fig. 14.

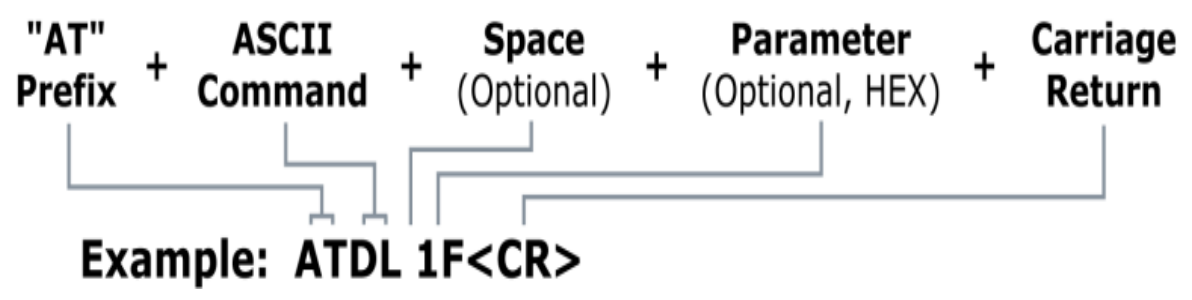

Figure 14. Syntax for sending AT commands

To establish communication between two XBee modules, the AT commands that are required to configure the modules are sequentially presented in Table 4. 
International Journal of Information Technology, Control and Automation (IJITCA) Vol.3, No.2, April 2013

\begin{tabular}{|l|l|}
\hline AT Commands & System Response \\
\hline+++ & OK $<\mathrm{CR}>($ Enter into Command Mode $)$ \\
\hline ATID(Pan ID) $<$ Enter $>$ & - \\
\hline ATMY $(16-$ bit Source Address $)<$ Enter $>$ & - \\
\hline ATDL $<$ Enter $>$ & $\{$ current value $\}<\mathrm{CR}>$ (Read Destination Address \\
\hline ATDL1A0D $<$ Enter $>$ & Low $)$ \\
\hline ATWR $<$ Enter $>$ & OK $<\mathrm{CR}>$ (Modify Destination Address Low) \\
\hline ATCN $<$ Enter $>$ & OK $<\mathrm{CR}>$ (Write to non-volatile memory) \\
\hline
\end{tabular}

Table 4. AT commands and responses for configuring XBee modules [13]

This communication system allows the user to send necessary commands to the copter. XBee modules are initialized with specific network ID and device number. "ATID", "ATMY", "ATDL" commands are used to set those ID and numbers. The sender and receiver modules are provided with the same network ID and specific device numbers for security purpose. The transmitted data are encrypted. Only the devices under same network ID and desired destination device number are able to decrypt the data. Thus security of the communication is ensured.

\section{System PROTOTYPE}

Working circuit is employed involving accelerometer and gyroscope to measure roll, pitch and yaw angles avoiding gimbal lock. Proximity sensor is employed to control elevation. Due to the unavailability of brushless DC motors in local market, low cost brushed DC motor is incorporated with gearing to 7:1 ratio to increase the loading capability sacrificing the speed. The frame built with Aluminum is lightweight but strong enough to hold the onboard circuits.

The wooden propellers (Fig. 15) are hand-made due to unavailability of ready-made propellers in the local market. Ready-made remotes are expensive and locally unavailable. So, a custom remote control system is designed and developed using XBee modules which offer faster and more reliable communication in long range. The resultant system is of low cost but enough to serve the purpose. The built frame for y4 copter is depicted in Fig. 16. Fig. 17 shows the on board control unit incorporating IMU and motor control unit and Fig. 18 demonstrates the custom remote built for communication between base and the copter. 


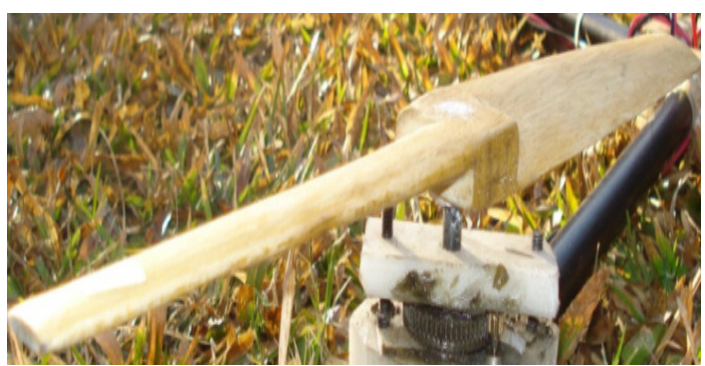

Figure 15. Wooden propeller designed for y4 copter

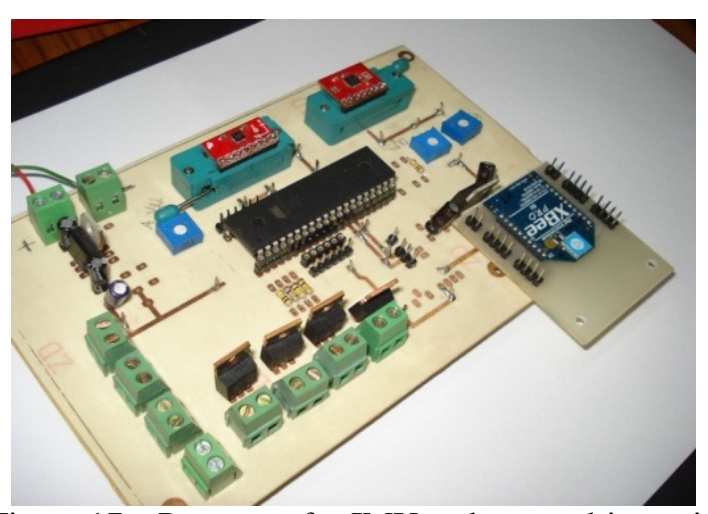

Figure 17. Prototype for IMU and motor drive unit

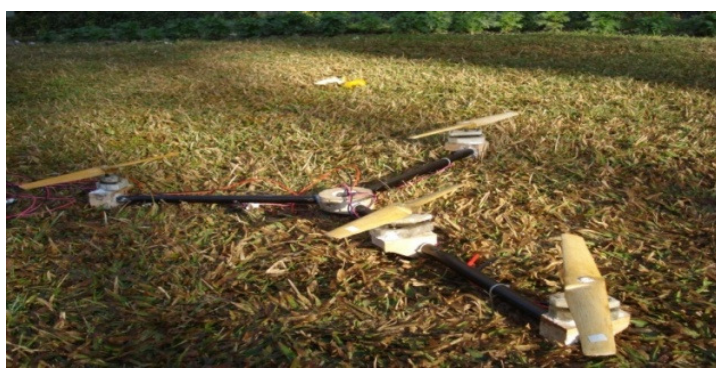

Figure 16. Built frame for Y4 copter

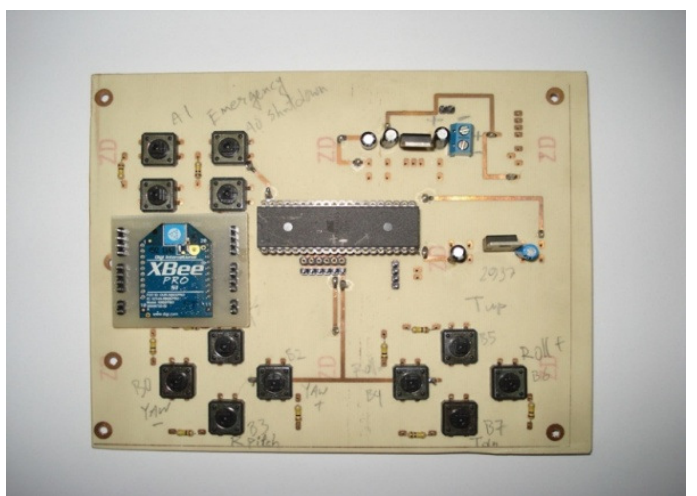

Figure 18. Custom remote using XBee

\section{SySTEM TESTING}

The system has been first tested in a closed room for safety issues. After several successful trial runs, the control system is developed with optimum $\mathrm{K}_{\mathrm{p}}, \mathrm{K}_{\mathrm{i}}$ and $\mathrm{K}_{\mathrm{d}}$. Firstly, their values have been determined using trial and error method and then corrected using Ziegler-Nichols method. The system is suitable for moderate environment. So, it has been tested for indoor flight only. Due to unavailability of batteries having high discharge rates with greater discharge time in local markets; the maximum flight time is no more than a few minutes. The self-stabilization and automatic landing systems work perfectly.

\section{FUTURE IMPROVEMENTS AND APPLICATIONS}

It has scope for further improvements. Mechanical strength can be improved for supporting outdoor flight. In future, high performance batteries will be imported to increase the flight time. Magnetometer will be used to develop IMU with 9 degrees of freedom for greater accuracy. Video surveillance with wireless video transmission is also a part of the plan. Wireless webcam is used to transmit video for a small area of coverage. This copter can easily be used in aerial photography, news collection, crime-scene investigation, geological exploration, fire-scene management, crowd control and for many other purposes. 
International Journal of Information Technology, Control and Automation (IJITCA) Vol.3, No.2, April 2013

\section{CONCLUSION}

An y4 copter with novel rotor configuration is designed and built successfully with custom Aluminum frame, wooden propellers and locally available low cost brushed DC motor without using costly ready-made components available on-line.

A unique self-stabilization system is designed for maintaining desired roll, pitch and yaw avoiding gimbal lock. Automatic elevation and landing is implemented using sonar equipment as proximity sensor. PID control scheme is implemented in an 8 bit microcontroller which has been proven just sufficient for control. Use of 8 bit microcontroller limits the limit of solvable complexity as it supports only the integer operations, but the operations are quite fast. Custom remote control system is developed using XBee modules ensuring 1 mile range of communication with security and reliability. Its built-in configurable 128 bit encryption system ensures secured communication between the base station and the copter. So, the implemented y4 copter is an automated aerial vehicle with effective self-stabilization system and automatic elevation and landing system with proper control scheme having a custom hand-built remote providing long range communication in a secured environment. This y4 copter with unique rotor configuration is the first reported y4 copter with fully autonomous sub-systems.

\section{REFERENCES}

[1] Md. Shafayat Hossain, Ariyan M. Kabir, Pratyai Mazumder, Ahmedullah Aziz, Masudul Hassan, Md. Azizul Islam and Pran Kanai Saha, "Design and Development of an Y4 Copter Control System," pp.251-256, 2012 UKSim $14^{\text {th }}$ International Conference on Modelling and Simulation, 2012.

[2] Dole, Charles E. Lewis, James E. "Flight Theory and Aerodynamics. A Practical Guide for Operational Safety, Second Edition." John Wiley and Sons, Inc. 2000.

[3] Bramwell, A.R.S. "Helicopter Dynamics". John Wiley and Sons, Inc.1976.Watkinson, John. "The Art of the Helicopter". Elsevier Butterworth-Heinmann. 2004.

[4] Sparkfun Electronics. "Triple-Axis Digital-Output Gyro ITG-3200 Breakout". Internet: http://www.sparkfun.com/products/9801, April 14, 2010 [Nov. 2, 2011].

[5] Sparkfun Electronics. "Triple Axis Accelerometer Breakout - BMA180". Internet: http://www.sparkfun.com/products/9723, April 15, 2010 [Nov. 2, 2011].

[6] J. B. Kuipers, "Quaternions and rotation Sequences: a Primer with Applications to Orbits, Aerospace, and Virtual Reality," Princeton University Press, 1999.

[7] Simon L. Altman, "Rotations, Quaternions, and Double Groups," Dover Publications, ch 12, 1986.

[8] Maxbotix. "LV-MaxSonar®-EZ0TM High Performance Sonar Range Finder." [On-line]. Pp. 1-2. Available: www.maxbotix.com/documents/MB1000_Datasheet.pdf [Jan. 2, 2012].

[9] Maxim. (2004, March). "MAX232 Datasheet." [On-line]. pp. 1-7. Available:www.datasheetcatalog.org/datasheet/texasinstruments/max232.pdf [Jan 3, 2012].

[10] Atmel. (2010, Oct. 20). “ATMEGA 32 datasheet." [On-line]. Pp. 1-233. Available: www.atmel.com/atmel/acrobat/doc2503.pdf [Nov. 1, 2011].

[11] J. B. Ziegler and N. B. Nichols, "Optimum Settings for Automatic Controllers," ASME Transactions, v64, pp. 759-768, 1942.

[12] Karl J. Åström \& Tore Hägglund, "Automatic Tuning of PID Controllers," Chapter 52, The Control Handbook, IEEE/CRC Press, 1995, William S. Levine ed.

[13] Sparkfun Electronics. "XBee Pro 60mW Chip Antenna - Series 1". Internet: http://www.sparkfun.com/products/8690, March 10, 2010 [Nov. 12, 2011]. 